\title{
Das Heldenkonzept des Räubers Karl Moor und der Räuber vom Liangschan-Moor sowie dessen Dekonstruktion bei Robert Walser
}

\author{
Fan Jieping
}

\section{Die Begrifflichkeit}

Literatur ist bekanntlich ein Terrain, wo Helden, Heldenideen und -konzepte konstruiert, entwickelt, transformiert und nicht zuletzt dekonstruiert werden, obwohl das Verständnis des Begriffs ,Held' im Westen und Osten unterschiedlich sein kann. Etymologisch gesehen stammte das englische Nomen hero vom griechischen heros, wobei heros in der griechischen Mythologie "Halbgott' meint. Als heros gelten etwa Herakles (Sohn des Zeus), Achilles (Sohn der Meeresnymphe Thetis). Es sind meist männliche Personen, die aus der Verbindung eines Gottes bzw. einer Göttin mit einem sterblichen Wesen hervorgegangen sind. Die Denotation dieses englischen Wortes hero lautet Ende des 14. Jahrhunderts etwa ,Mann von übermenschlicher Stärke oder körperlichem Mut'.

Das deutsche Wort ,Held' geht auf das 9. Jahrhundert zurück. Das mittelhochdeutsche helt und helid bedeutet ,Kämpfer und freier Mann'. ${ }^{1}$ Mir scheint der Hinweis relevant zu sein, dass das Wort ,Held' einen gewissen Zusammenhang zu $k^{w} e l$ in den indogermanischen Sprachen habe, dessen Bedeutungselement wie ,besorgen' zu den deutschen Wörtern ,Wirt und Bauer' geführt habe. ${ }^{2}$ Das Nomen ,Held“ konnotiert überdies ,harte Haut'. Dagegen ist der abgeschwächte Gebrauch von ,Held“ als bloße ,Hauptperson' in der Literatur eine Lehnbedeutung nach dem Neuenglischen hero. ${ }^{3}$ Die moderne Bedeutung von ,Held' als ,Mann, der große Tapferkeit zeigt und sich in den Dienst der Menschheit stellt', findet sich bereits seit dem 15. Jahrhundert in den indogermanischen Sprachen. Im 18. Jahrhundert gewann die Heldenverehrung einen Aufschwung durch den zunehmenden Rückbezug auf die antike Heroik.

Im Chinesischen hat das Wort ,Held‘ eine Entsprechung, die yingxiong (英雄) lautet. Der Begriff ,Held', also das Wort yingxiong, besteht aus zwei chinesischen Zeichen. Vor dem Jahre 221 (v. Chr.) wurden die beiden Zeichen allerdings getrennt verwendet, nämlich das ying für ,Blume und Blüte` und das xiong für ,männ-

1 Friedrich Kluge: Etymologisches Wörterbuch der deutschen Sprache, bearbeitet von Elmar Seebold, Berlin/New York ${ }^{23} 1995$, S. 368.

2 Ebd.

3 Ebd. In der deutschen Literatur gewann das Nomen ,Held‘ die Bedeutung als ,männliche Hauptfigur' in einem Bühnenstück und in einem Roman erst zu Beginn des 17. Jahrhunderts. 
liches Wesen'. Das Wort yingxiong als Sammelbegriff für ,Held‘ kam erst Ende der Südlichen Han-Dynastie (200 n. Chr.) oder genauer in der Zeit der Drei Reiche (208-280 n. Chr.), also vor ca. 2000 Jahren, in den chinesischen Geschichtsbüchern auf, nämlich in dem Buch Hanmo Yingxiong Ji (《汉末英雄记》). ${ }^{4}$ Dort wurde das Heldenkonzept Chinas mit affirmativer Attitüde zum kriegerischen, gewaltsamen, kultischen und charismatischen Charakter erstmals in der Zeit der späteren Südlichen Han-Dynastie konstruiert.

\section{Heldenkonzepte}

In der europäischen Literatur ist die Konstruktion, Transformation und Dekonstruktion des Heldenkonzeptes ein langer Prozess, der von unterschiedlichen Faktoren beeinflusst wird. Die Genese des literarischen Heldenkonzeptes lässt sich am deutlichsten auf den Einfluss der antiken Mythologie zurückführen. Ein besonderes Phänomen macht hierbei aufmerksam. Die griechischen Heroen (ท๊ $\rho \omega \varepsilon \varsigma)$ waren häufig die männlichen Gestalten, die als mythische Gründer der griechischen Städte, Staaten und Länder galten. Sie sind übermenschlich stark, tapfer, aber auch zum Selbstopfer bereit wie Prometheus. Während diese mythischen Helden ausschließlich Kämpfer mit besonderer andreia ( $\dot{\alpha} v \delta \rho \varepsilon i ́ \alpha)$, also maßgeblich maskulin konstruiert waren, kam den weiblichen Personen oft nur eine Nebenrolle zu. Aber die mythischen Helden waren nicht immer tadellose Vorbilder, selbst der unverwundbare Held Achilleus hat auch eine Schwäche: Seine Ferse war die einzige Stelle, an welcher er verwundbar war.

Um die Konstruktion eines Heldenkonzeptes in der europäischen Literatur zu untersuchen, lässt sich an die internationale aktuelle Herologieforschung ${ }^{5}$ anknüpfen: Das literarische Heldenkonzept ist demnach erstens ein kulturelles Konstrukt, das im Rahmen des jeweiligen kulturellen Kontexts zu betrachten ist. Zweitens ist der kultisch verehrte Held ein Konstrukt der gesellschaftlichen Institutionen und Ideologien. Drittens ist dieses Konstrukt nur durch eine bestimmte Medialisierung möglich.

Das Heroische manifestiert sich in heroischen Figuren und ist als kulturelles Konstrukt ein Fremd- und Selbstzuschreibungsphänomen. In seiner kultur-, gruppen- und zeitspezifischen Prägung entzieht es sich essentialistischen Bestimmungen. ${ }^{6}$

4 Das Buch Hanmo Yingxiong Ji (Die Geschichte der Helden Ende der Han-Dynastie) in 10 Bänden wurde vom Gelehrten und Dichter der Südlichen Han-Dynastie Wang Can (王粲 177-217 n. Chr.) im Jahre 208 n. Chr. vollendet. Dieses Helden-Buch war aber vollständig verloren gegangen. Die Überlieferung der Heldengeschichten geht auf Adaptionen anderer Geschichtsbücher zurück.

5 DFG-Sonderforschungsbereich (SFB) 948 „Helden - Heroisierungen - Heroismen. Transformationen und Konjunkturen von der Antike bis zur Moderne“ an der Albert-LudwigsUniversität Freiburg i. Br.

6 Ralf von den Hoff u.a.: Helden - Heroisierungen - Heroismen. Transformationen und Konjunkturen von der Antike bis zur Moderne. Konzeptionelle Ausgangspunkte des Son- 
[...] Als heroische Figur verstehen wir [...] zunächst eine reale oder fiktive, lebende oder tote menschliche Person, die als Held, hero, héros usw. benannt und/oder präsentiert wird und der heroische Eigenschaften zugeschrieben werden, und zwar insbesondere agonale, außeralltägliche, oftmals transgressive eigene Leistungen. ${ }^{7}$

[...] Über eine heroische Figur wird in unterschiedlichen, auch künstlerischen Medien berichtet, sie besitzt charismatische Wirkung und sie wird vor allem von einer Gemeinschaft ,gefolgschaftlich'v verehrt [...]. Gleichwohl teilt auch die heroische Figur manche grundlegende körperliche und emotionale Eigenschaft, aber auch Handlungs- und Leidensfähigkeit mit ihren Verehrer/innen. ${ }^{8}$

Demnach äußert sich ein Heldenkonzept, zumindest in der Literatur, in Gestalt eines Helden, der von den je zeitspezifischen kulturellen und medialen Einflüssen geprägt ist. Nicht selten sind literarische Figuren so konstruiert, dass sie nach bestimmten Wertvorstellungen sogar als Volks- oder Nationalhelden gestaltet sind. Dieses Phänomen hängt vom jeweiligen gesellschaftlichen Bewusstsein ab, das Autor und Leser bestimmt. So werden in der antiken Mythologie den Helden häufig besondere Kräfte und eine übertriebene Maskulinität zugeschrieben. Zum ,Helden wird ein Mensch durch eine außergewöhnliche (,heroische') Tat, wenn er etwa einen Feind, ein Ungeheuer oder einen Riesen erschlägt, eine Blutrache ausführt oder Menschen aus Bedrängnis rettet. Die Heldentat ist wiederum durch die sozialen und kulturellen Konstruktionen von Männlichkeiten und Weiblichkeiten charakterisiert. Im westlichen Heldenkonzept dominiert überdies ein ausgeprägter Individualismus bzw. ein starker Willen nach Freiheit.

Dagegen werden in chinesischen Geschichtsbüchern oft diejenigen als ,Helden“ bezeichnet, die dem Kaiser ihre Loyalität durch Tapferkeit in Kriegen erwiesen, auch wenn sie vom Kaiser missverstanden und verfolgt wurden. General Yue Fei (岳飞) und Wen Tianxiang (文天祥) 9 sind typische Beispiele dafür. Sie waren oft Persönlichkeiten, „die einen sehr weiten Horizont besitzen und über außergewöhnliche Kompetenzen verfügen, sie erlangen ihren Ruhm durch ihre tapfere Taten“" ${ }^{10}$ Das Heroische in der chinesischen Kultur ist wie im Heldenkonzept des Westens derart konstruiert, dass einem Menschen edle Tugend, Opferbereitschaft, ausge-

derforschungsbereichs 948, in: helden. heroes. héros. E-Journal zu Kulturen des Heroischen 1.1, 2013, S. 7-13, hier S. 8. DOI: 10.6094/helden.heroes.heros./2013/01/03.

9 Yue Fei (1103-1142) war chinesischer Militärgeneral in der Südlichen Song-Dynastie. Er ist dafür bekannt, dass er im 12. Jahrhundert in den Kriegen zwischen der Südlichen Song- und der von Jurchens regierten Jin-Dynastie in Nordchina die Streitkräfte der Südlichen Song führte, bevor er 1142 vom Kaiserhof wegen seiner vermeintlichen Schulden und schließlich unter einer zusammengebrachten Anklage getötet wurde. Wen Tianxiang (1236-1283) war in den letzten Jahren der Südlichen Song-Dynastie ein Generalwissenschaftler. Für seinen Widerstand gegen Kublai Khans Invasion und seine Weigerung, der Yuan-Dynastie nachzugeben, wurde er ermordet. Yue Fei und Wen Tianxiang gelten in China als Volkshelden.

10 Vgl. Lei Min: Historical and Cultural Connotation of Chinese and Western National Heroes' Loyalism: Romance of the Three Kingdoms and Greek Mythology, in: CrossCultural Communication 5.2, 2014, S. 134-140, hier S. 90 (übers. von Verf.). 
wöhnliche Taten und fast übermenschliche Kampfkunst wie Kungfu zugeschrieben werden. Der Unterschied besteht darin, dass „ein Held in der chinesischen Tradition dem konfuzianischen Moralkanon, also der Familie und ihrer Sippe sowie bestimmten gesellschaftlichen Wertvorstellungen untergeordnet " ist. ${ }^{11}$

Das Gemeinsame in den literarischen Heldenkonzepten - sowohl im Westen als auch in China - besteht darin, dass Helden als künstlerische Konstrukte mit kultur- und zeitspezifischer Prägung zu verstehen sind. Auf der einen Seite kämpfen sie für die Freiheit und Gerechtigkeit, die „eine Mitte, freilich nicht auf dieselbe Art wie die übrigen Tugenden, sondern weil sie die Mitte schafft“. ${ }^{12}$ Dafür greifen sie zur Waffe und Gewalt. Auf der anderen Seite werden das männliche Geschlecht und dessen Männlichkeit bei der Heroisierung bevorzugt. Selbst wenn Frauengestalten als ,Heldinnen ' überhaupt vorkommen, wird ihnen - jedenfalls in der chinesischen Literatur - ausnahmenlos eine gewisse Maskulinität zugeschrieben: Entweder sind sie in Kriegen als Mann verkleidet oder verfügen wie die Männer über außergewöhnliche Kampfkunst und sind unbesiegbar. In der Ballade von Mulan (《木兰辞》) $)^{13}$ und Die Legende der Generäle der Familie Yang (《杨家 将演义》) $)^{14}$ sind die Heldinnen Hua Mulan (花木兰) und Mu Guiying (穆桂英) durch Zuschreibung ,männlicher ${ }^{6}$ Eigenschaften heroisiert. ${ }^{15}$

Die Heroisierungen literarischer Figuren haben in der Weltliteratur eine lange Tradition. Sie verlaufen aber keineswegs immer gleich, nicht statisch, sondern dynamisch, da sie in einem Relationengefüge zu sehen sind, wie Helmuth Plessner zu Recht hervorhebt: „Die Qualitäten, die einer heroischen Figur zugeschrieben werden, sind variabel. Den Vorgang der Zuschreibung, an dem unterschiedliche Akteure beteiligt sind und durch den die Figur zum gestalthaften Fokus einer Gemeinschaft wird, nennen wir Heroisierung". ${ }^{16}$

Heroisierungen vollziehen und stabilisieren sich also in sozialen und kommunikativen Prozessen, die medialer Präsentation bedürfen und affektiv wie normativ aufgeladen sind. In diesem Sinne ist die Literatur eines der relevantesten Medien, in dem Heroisierungen präsentiert werden. Um diese These zu erhärten, werden in

11 Ebd., S. 91.

12 Aristoteles: Nikomachische Ethik, Berlin 2014, 1133 b 32.

13 Die Ballade $M u$ Lan wurde im 9. Jahrhundert n. Chr. in den Sammelband Yuefu Gedichte (《乐府诗集》) aufgenommen.

14 Die Urform von Die Legende der Generälenfamilie Yang war der Helden-Roman Die Geschichten der Nördlichen Song-Dynastie (《北宋志传》) von Xiong Damu (熊大木) im 14. Jahrhundert n. Chr.

15 Die beiden waren legendäre Kriegerinnen aus dem traditionellen China. Es wurde in der Ballade von Mulan beschrieben, dass Hua Mulan, als Mann verkleidet, für ihren Vater zwölf Jahre lang im Krieg gegen Hunnen kämpfte. Mu Guiying ist eine Generalin im Krieg gegen Mongolen im 8. Jahrhundert n. Chr. Sie beherrschte eine hervorragende Kampfkunst und war Mitglied der Generalsfamilie Yang in der Song-Dynastie. Vgl. auch den Beitrag von Stefanie Lethbridge zur filmischen Umsetzung dieser martialischen Heldinnen im postmodernen Film in diesem Sammelband.

16 Helmuth Plessner: Macht und menschliche Natur, Frankfurt am Main 1981, S. 48. 
der folgenden Fallstudie Karl Moor in Friedrich Schillers Schauspiel Die Räuber, die Räuber im klassischen chinesischen Roman Shuibu Zhuan (《水湤传》) von Shi Naian (施耐庵) ${ }^{17}$ und der Räuber in Robert Walsers Romanfragment Räuber verglichen, um zu erläutern, wie der Typus ,Räuber' als Held in der deutschen und chinesischen Literatur konstruiert, heroisiert und deheroisiert wird und welche Unterschiede und Gemeinsamkeit festzustellen sind. Die spezifischen Ausformungen der Heroisierungsprozesse hängen von den beteiligten Akteuren und ihren Motivationen ab. Es stellt sich zudem die Frage, in welcher Form und warum das Heroische als Zuschreibung überhaupt genutzt und wirksam wird.

\section{Schillers Karl Moor als Räuber}

Schillers Drama Die Räuber ist 1781 in der literarischen Epoche des Sturm und Drang entstanden. Schiller wollte offensichtlich mit diesem Drama - konkret mit der Figur Karl Moor - den Konflikt zwischen Verstand und Gefühl, Freiheit und Justiz darstellen. Seinem Heldenkonzept entspricht Karl Moor, der als Hauptmann einer Räuberbande in böhmischen Wäldern von seiner Gefolgschaft verehrt wird. Als Held ist er nicht nur kraftvoll, kompromisslos, tapfer und gewaltsam, sondern auch intelligent, edel und melancholisch. Er hat menschliche Schwächen, wie sie alle Helden in der westlichen Literatur haben. Schiller hat ihn als Held konstruiert, um mit dem Zeitgeist ein Originalgenie - ein großes, unabhängiges Individuum in der Räuberfigur Karl Moor zum Ausdruck zu bringen. ${ }^{18}$

In der dritten Szene des zweiten Aktes bekennt Karl Moor dem Pater gegenüber, der die umzingelte Räuberbande im Wald zur Auslieferung ihres Hauptmannes zu bewegen versucht:

Wahr ist's, ich habe den Reichsgrafen erschlagen, die Dominicuskirche angezündet und geplündert, hab' Feuerbrände in eure bigotte Stadt geworfen und den Pulverthurm über die Häupter guter Christen herabgestürzt. ${ }^{19}$

Zwar bekennt sich hier Karl Moor einerseits zu seinen Gewalttaten, die er zur Rettung seines Miträubers Roller begangen hat, betont aber zugleich, dass er nur Gewalt gegen die Gewalt geübt habe, weil die Stadt Roller gefangen genommen und zum Tod verurteilt hat. Andererseits wendet er aber keine Gewalt gegen den Pater an, indem er die Vorschläge Rollers und Schweizers ablehnt: (Schweizer:) „Soll ich

17 Deutsche Übersetzung von Franz Kuhn: Die Räuber vom Liang-Schan-Moor, Leipzig 1934; englische Übersetzung von Pearl S. Buck: All Men Are Brothers, New York 1933.

18 Schillers Heldentum kommt in seiner Ode An die Freude im Jahr 1785 zum Ausdruck. Darin werden die auf das männliche Geschlecht bezogene Brüderlichkeit und der kämpferische Geist für die Gerechtigkeit hervorgehoben. Schiller schreibt in der ersten Strophe: „Bettler werden Fürstenbrüder“ (geändert zu: „Alle Menschen werden Brüder“ in der 2. Fassung 1808) und in der neunten Strophe: „Rettung von Tyrannenketten“. www.friedrichschiller-archiv.de/gedichte-schillers/highlights/an-die-freude/, 23. Januar 2020.

19 Friedrich Schiller: Die Räuber, Leipzig 1905, S. 105 (2. Akt, 3. Szene). 
hingehn und diesem abgerichteten Schäferhund die Gurgel zusammenschnüren, daß ihm der rote Saft aus allen Schweißlöchern sprudelt?" 20 oder (Roller:) „Soll ich diesen Kerl das oberst zu unterst unters Firmament wie einen Kegel aufsetzen? ‘21 Was Karl Moor hier zu rechtfertigen beabsichtigt, ist nichts anderes als eine radikale Umsetzung der Heldenidee des Dichters Friedrich Schiller, die aus dem Fortgang der Rede des Räubers Karl Moor deutlich wird:

Bemerken Sie die vier kostbaren Ringe, die ich an jedem Finger trage? [...] [D]iesen Rubin zog ich einem Minister vom Finger [...]. Er hatte sich aus dem Pöbelstaub zu seinem ersten Günstling emporgeschmeichelt, der Fall seines Nachbars war seiner Hoheit Schemel - Thränen der Waisen huben ihn auf. Diesen Demant zog ich einem Finanzrath ab, der Ehrenstellen und Ämter an die Meistbietenden verkaufte und nicht trauernden Patrioten von seiner Thüre stieß. - Diesen Achat trag' ich einem Pfaffen Ihres Gelichters zur Ehre, den ich mit eigener Hand erwürgte, als er auf offener Kanzel geweint hatte, daß die Inquisition so in Zerfall käme [...]. ${ }^{22}$

In dieser Rede wird Karl Moor heroisiert, indem Schiller ihn nicht nur den Fürsten und den Finanzrat als Vertreter der Aristokratie, den Pfarrer als Repräsentanten der christlichen Kirche entlarven lässt, sondern auch dadurch seine brutale Gewaltanwendung legitimiert. Mit dem Satz: „Pfui! Pfui über das schlappe Kastratenjahrhundert" ${ }^{\text {"23 }}$ wird die Maskulinität des Helden implizit betont. Denn Karl Moor wird als Hauptmann von seiner verbrüderten Gefolgschaft einerseits verehrt, andererseits verlangt seine Autorität von ihm, eine gewisse Verantwortung für seine Brüder zu übernehmen. Er ist ein kühner Kämpfer, doch auch der nachdenkliche und melancholische Träumer; er ist ein harter, durch nichts zu erschreckender Mann, doch auch der Gefühlsmensch. Auf der einen Seite seine verbrüderte Räuberbande, auf der anderen Seite seine Geliebte Amalia und Adelsfamilie. Dieses Dilemma lässt sich als persönliche Schwäche eines Helden verstehen. Das transformierte Heldenkonzept bei Schiller hat zur Folge, dass sein Held Karl Moor immer wieder zwischen der Wiederherstellung der alten Ordnung und einer konsequenten Ablehnung des „Kastratenjahrhunderts“24 schwankt. Seine Inkonsequenz führt schließlich dazu, dass er seine Bandenmitglieder verrät und sich selbst der Justiz ausliefert.

\section{Die Räuber vom Liangschan-Moor}

Der Roman Shuibu Zbuan ist einer der vier chinesischen Romanklassiker, der in der Song-Dynastie im 14. Jahrhundert entstanden ist. Dieses literarische Werk schildert in Form eines spannenden Abenteuerromans die Rebellion einer Gruppe legendä-

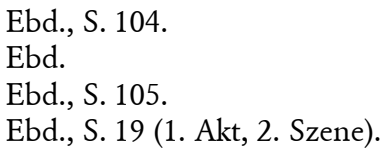


rer Helden, die die Reichen bestehlen und die Armen beschenken. Klaus Mühlhahn hat 1992 festgestellt, dass alle Räuber-Figuren durch eine akzentuierte Beschreibung der Maskulinität heroisiert seien. ${ }^{25} \mathrm{Er}$ hat obendrein eine Frage zum Heldenkonzept des Romans gestellt, ob die ,Räuber vom Liangschan-Moor ${ }^{6}$ wirklich im traditionellen Sinne ,Helden' seien. Mühlhahn ordnet sie eher einer Zwischenkategorie von Rittern, Räubern und Helden zu. Auch in China wird oft diskutiert, ob sie als Volkshelden bezeichnet werden sollen, weil viele von ihnen, bevor sie sich auf dem Liangschan-Moor sammelten, gewalttätig und bösartig seien. Viele von ihnen seien Diebe, Banditen, Schurken, Kannibalen gewesen, sie seien gewalttätig gegen die korrupten kaiserlichen Beamten, aber auch gegen die Zivilisten. ${ }^{26}$ Ein solcher konfuser Diskurs lässt sich auf ein unscharfes Heldenkonzept zurückführen.

Die 108 Räubergestalten des Romans werden von Shi Naian so dargestellt, dass sie sehr unterschiedliche Charaktere und Eigenschaften haben. Auch ihre jeweilige Herkunft ist ganz uneinheitlich und differenziert. Die im Folgenden zu diskutierenden 36 Haupträuber ${ }^{27}$ werden alle wegen sozialer Ungerechtigkeit und Unterdrückung auf das Liangschan-Moor gezwungen, wie es bei Schillers Räubern in den böhmischen Wäldern der Fall ist. Sie waren vorher etwa Lehrer, Unteroffiziere, Beamte, Kaufleute oder Bauern. ${ }^{28}$

Die Räuber vom Liangschan-Moor scheinen dem Heldenkonzept der chinesischen Heldenliteratur entsprechend heroisiert. Zunächst hat der Autor seine Räuber - die 36 Haupträuber - derart konstruiert, dass sie alle ordnungsgemäß im Rahmen des konfuzianischen Moralkanon lebten. Nachdem sie Intrigen und Missgunst des Kaiserhofes zum Opfer gefallen waren, versammelten sich alle schließlich um den Anführer einer Rebellenarmee namens Song Jiang (宋江). Zweitens verfügen sie alle über besondere Fähigkeiten, vor allem übermenschliche Kraft und hervorragende Kampfkunst. Drittens waren sie alle Verfolgte und Geächtete, da sie gesellschaftliche Vorbilder waren, bevor sie zur Räuberei gezwungen wurden. Nicht zuletzt sind sie mit einer gewissen Affinität zur Unterschicht der Bevölkerung konstruiert. Die im Roman durchgängig hervorgehobene Brüderlichkeit und Maskulinität sind ein besonderes Merkmal bei der Heroisierung. Auch die Trunksucht und Vorazität spielen eine wichtige Rolle. Der ehemalige Unteroffizier Wu Song (武松) verfügt zum Beispiel über außergewöhnliche Kraft und hat sogar in Berauschtheit

25 Klaus Mühlhahn: Ritter, Räuber, Helden, Bilder und Ansichten der Männlichkeit im Shuibuzhuan, in: Das neue China, Berlin 1992, S. 13-15.

26 Vgl. Feng Ruchang: The Hero's Beginning and Destination of „Out-laws of the Marsh“, in: Journal of University of South China (Social Science Edition) 6, 2009 (auf Chinesisch), S. 89-92, hier: S. 90 (übers. von Verf.).

27 Die 108 Räuber werden nach der daoistischen Astrologie in zwei Gruppen unterteilt. Die 36 Haupträuber werden als „Glückssterne“ (天罡星) und die 72 Nebenräuber als „Unglückssterne“ (地繁星) kategorisiert.

28 Bei Schiller wird die Herkunft der Räuber so geschildert: „und jetzt sind unserer acht und siebenzig, meistens ruinierte Krämer, rejicierte Magister und Schreiber aus den schwäbischen Provinzen." (Schiller: Die Räuber [Anm. 19], 3. Akt, 2. Szene, S. 19). 
einen Tiger mit bloßen Fäusten totgeschlagen. Der Kungfu-Lehrer der kaiserlichen Armee Lin Chong (林冲) beherrscht grandiose Kampfkunst; Lu Da (鲁智深), auch ein Unteroffizier und später ein buddhistischer Mönch, kann im betrunkenen $\mathrm{Zu}$ stand einen riesigen Weidenbaum allein aus der Erde herausreißen.

Die Heroisierung der Räuber vom Liangschan-Moor findet hauptsächlich in den ersten siebzig Kapiteln des Romans statt. Weniger vom Streben nach individueller Freiheit und persönlicher Ehre wie Karl Moor geleitet, kämpfen die Räuber vom Liangschan-Moor vielmehr um die soziale Gerechtigkeit und Brüderlichkeit. Das Heroische scheint hierbei nichts anderes als verschiedenartige Performanz des Kampfes für die soziale Gerechtigkeit zu sein. Auch wenn Straftaten begangen werden, handelt es sich meistens darum, dem Schwächeren zu helfen, etwa nach dem Motto: Ein Held soll eine Lanze für jeden brechen, der ungerecht behandelt wird.

Genauso wie Karl Moor ist Song Jiang, der Anführer der Rebellenarmee, beim Kampf gegen den Kaiserhof und gegen die soziale Ungerechtigkeit allerdings inkonsequent. Im Schlusskapitel des Romans schlägt die Heroisierung aber in „Loyalität“" gegenüber dem Kaiser um. Dies wird im 71. Kapitel folgendermaßen dargestellt: Nach einer Feier im „Saal der Loyalität und Brüderlichkeit“ ${ }^{29}$ schwören die Räuber, dass sie „auf Leben und Tod und für die Ewigkeit ungetrennt wie heute zusammenbleiben “. ${ }^{30}$ Lu Junyi (卢俊义), ein wichtiger Räuberführer neben Song Jiang, ist betrunken und träumt, der Kaiser hätte einen General geschickt, um ihn festzunehmen. Song Jiang hätte eine Strategie ausgedacht, zu kapitulieren, damit alle Räuber vom Kaiser amnestiert würden. Lus Alptraum endet damit, dass alle 108 Räuber trotzdem geköpft werden. Denn der Kaiser besteht darauf, dass nur dadurch die „Stabilität des Kaiserreichs“31 gesichert werden könne. Während das Heldenkonzept beim Räuber Karl Moor deutlich von der schwankenden Anerkennung des alten, patriarchalisch bestimmten Ordnungsgefüges in der Welt und der Gottheit als letzter Instanz geprägt ist, dominiert bei Song Jiang untergründig die Loyalität zum Kaiserhof und zur feudalistischen Ordnung.

\section{Dekonstruktion des Heldenkonzeptes im Räuber-Romanfragment}

Das traditionelle Subjektkonzept, und damit das literarische Heldenkonzept, wurde im Zuge der Neugestaltung der Wissensordnung bzw. im Prozess der Entmythologisierung seit der Neuzeit immer wieder in Frage gestellt. Das herkömmliche Heldenkonzept wurde in Theorie wie Praxis stets transformiert und gar dekonstru-

29 Shi Naian: Shuihu Zhuan, Shanghai 1944 (auf Chinesisch), S. 473 (übers. von Verf.).

30 Ebd.

31 Ebd., S. 474. 
iert. ${ }^{32}$ Nora Weinelt hat die Konstruktion des Heldenkonzeptes sowohl im fiktiven als auch im realen Kontext folgendermaßen zusammengefasst:

Erstens: Der Held wird Held nur, indem er handelt. Zweitens: Er tut dies im Rahmen der jeweils prävalenten (das heißt vorherrschenden) gesellschaftlichen Ideale und Ideologien in irgendeiner Form moralisch sinnhaft, seine Tat folgt einer edlen Absicht. Und drittens: Der Held hebt sich in seinem Handeln deutlich vom Rest der Bevölkerung ab; er steht als liminale und potentiell transgressive Figur am Rande der Gesellschaft. ${ }^{33}$

Inwieweit bestimmt die moderne Transformation des Heroischen auch Robert Walsers Romanfragment Räuber? Dieses literarische Meisterwerk ist etwa im Juli und August 1925 in Bern entstanden. Der Roman wurde von Walser als mikrographischer Bleistiftentwurf auf 24 Manuskriptblättern geschrieben. Er ist neben den drei Berliner Romanen Walsers umfangreichster Text und gilt als ein zentraler Bestandteil seines literarischen Werks, obwohl keine autorisierte Reinschrift überliefert ist.

Allerdings hat das Roman-Manuskript einen engen Zusammenhang mit Schillers Räubern. So hat Bernhard Echte darauf hingewiesen, dass Walser versucht haben soll, seine Kinderheitserinnerung eines Theaterbesuchs von Schillers Räuber 1894 in Biel in Form eines Romans zu schreiben. ${ }^{34}$ So behauptet der Ich-Erzähler des Romans, dass ein „Aquarell-Bildchen, das ein jugendlicher, kaum dem Knabenalter entwachsener Maler ausführte, [...] zu all diesen kulturellen Zeilen den Anlass" gab. ${ }^{35}$ Das hier genannte Aquarell-Bildchen ist in der Tat ein Bild, das Walsers Bruder Karl 1894 gemalt hatte und das Walser jahrzehntelang bei sich behalten hat. Auf dem Bild ist zu sehen:

Der junge Frohmutige bekleidet sich mit einer Samtweste, die sein Vater zu Hochzeiten getragen hat. Über die Schulter wirft er einen alten Onkelsmantel, der in einer Stadt am Mississippi erhandelt worden ist, und um die Hüften wird eine Glarnerschärpe gezwungen. Der Kopf bekommt eine zweckentsprechende Bedeckung [...]. Die Hand hat sich eine gräuliche Pistole zu verschaffen gewusst, und den Beinen haften Wildhüterstiefel an. Also ausstaffiert wird „Karl“ eingeübt. ${ }^{36}$

Ist dies ein Heldenbild von Robert Walsers Räuber? Ist es etwa eine gewaltige, übermenschlich starke, tapfere und virile Helden-Figur, die Robert Walser in sei-

32 Vgl. Fan Jieping: Robert Walser and Criticism on Subject Discourse, Hangzhou 2011 (auf Chinesisch); Fan Jieping (Hg.): Subject Discourse, Practice and Criticism, Hangzhou 2017 (auf Chinesisch).

33 Nora Weinelt: Zum dialektischen Verhältnis der Begriffe Held und Antiheld - Eine Annäherung aus literaturwissenschaftlicher Perspektive, in: helden. heroes. héros. E-Journal zu Kulturen des Heroischen 3.1, 2015, S. 15-22, hier S. 16. DOI: 10.6094/helden.heroes. heros/2015/01/03.

34 Bernhard Echte (Hg.): Robert Walser. Sein Leben in Bildern und Texten, Frankfurt am Main 2008, S. 38-39.

35 Robert Walser: Räuber-Roman, in: Aus dem Bleistiftgebiet, Bd. 3, Räuber-Roman, FelixSzenen, hg. von Bernhard Echte und Werner Morlang, Frankfurt am Main 1986, S. 11-149, hier S. 148.

36 Ders.: Wenzel, in: Sämtliche Werke, Bd. 2, Frankfurt am Main 1986, S. 81-91, hier S. 84. 
nem Räuber-Roman gestalten will? Die Antwort ist eindeutig. Denn der IchErzähler oder der Räuber des Romanfragments antwortet selbst darauf, als er eines Abends „ein großes Beethovenkonzert hörte“, und eine „Fürstin [...] im Konzertsaal neben [ihm] “ saß ${ }^{37}$ und zu ihm spricht: „Du bist ein Feind der Allgemeinheit. Du schuldest mir Zärtlichkeit. [...] Ich sehe dir an, dass du Ehemannstugenden hast. Mir scheint, du hast einen starken Rücken. Deine Schultern sind breit." 38 Der Räuber bestreitet das, indem er mit leiser Stimme vorbringt: „Meine Achseln sind das Zarteste, was je in dieser Hinsicht geschaffen worden ist.“ - „Du bist ein Herkules.“ - „Das scheint nur so.“39 Anschließend schildert Robert Walser die Heldenpose seines Räubers oder seiner selbst:

Und so ein Ausreißer ging im Räuberkostüm umher. Er trug einen Dolch im Gürtel. Die Hose war breit und mattblau. Eine Schärpe hing ihm am schmalen Leib. Hut und Haar vergegenwärtigten das Prinzip der Unerschrockenheit. Das Hemd schmückte ein Spitzenbesatz. Der Mantel war allerdings etwas fadenscheinig, immerhin aber mit Pelz verbrämt. Die Farbe dieses Ausstattungsstückes war ein nicht allzu grünes Grün. [...] Die Pistole, die er in der Hand hielt, lachte über ihren Besitzer. ${ }^{40}$

Im Gegensatz zu Schillers Karl Moor ist der Räuber bei Robert Walser eher ein Fliehender, der sich „,vor der Frau in Braun wie ein Mädchen“41 verhält. Im Vergleich zur Maskulinität und Gewaltlust der Räuber in böhmischen Wäldern und der Räuber vom Liangschan-Moor hebt Walser bei seinem Räuber dagegen eine Affinität zur Weiblichkeit und Zärtlichkeit hervor. Walser betont: „[...] vielleicht zeitweise [war] der Räuber wirklich ein Mädchen, so eine Art Mägdlein geworden." ${ }^{42}$ Es scheint so, dass der Räuber in der erfolgssüchtigen Männerwelt nichts zu suchen hat. Denn ,in eine Magd also wurde er verwandelt. Es scheint, dass er in einer Schürze umherlief, und es scheint zugleich, dass er sich dieses lieblichen Schmucks aufrichtig freute. "“3 Beinah alle nennenswerten Nebenfiguren im Räuber-Roman sind ausschließlich Frauen, bei denen Walsers Räuber seine Identität zu finden versucht. Etwa die Kennerin Edith, die ihn liebt, und ihm zugleich wegen angeblich versprochener und nicht gezahlter 100 Franken nachstellt; die Bürgertochter Wanda, die er liebt und durch seine Werbungen bloßstellt; die Witwe, deren Kaffee-Löffelchen er heimlich ableckt, usw. Kurzum: „Hunderte von Unterröckchen sympathisieren mit ihm." ${ }^{44}$ Es geht einmal sogar so weit, dass der Räuber zum Arzt geht, bei dem er sich darüber beschwert:

37 Ders.: Räuber-Roman (Anm. 35), S. 19.

38 Ebd., S. 19-20.

39 Ebd., S. 20.

40 Ebd.

41 Ebd., S. 19.

42 Ebd., S. 109.

43 Ebd., S. 108-109.

44 Klaus-Michael Hinz / Thomas Horst (Hg.): Robert Walser, Frankfurt am Main 1991, S. 225. 
Ich bekenne Ihnen ohne Umschweife, daß ich mich dann und wann als Mädchen fühle [...], hochverehrter Herr, daß ich ganz fest glaube, ich sei ein Mann wie irgendein anderer, nur daß mir oft schon, [...] daß ich gar keine Angriffs-, keine Besitzlust in mir lodern, weben und aus mir herausdrängen spüre. Im übrigen halte ich mich für einen ganz braven wackeren Mann, für einen durchaus brauchbaren Mann. Ich bin arbeitslustig, ohne dass ich allerdings zur Zeit viel leiste. [...] Ich glaube, es lebe vielleicht in mir eine Art von Kind oder eine Art von Knabe. [...] Für ein Mädchen hielt ich mich ein paar$\mathrm{mal}[\ldots]{ }^{45}$

An dieser Passage wie an vielen anderen im Räuber-Roman lässt sich erkennen, dass Robert Walser offensichtlich eine andere Heldenidee hat, die dem traditionellen Heldenkonzept widerspricht. Er hat in seinem Räuber eine Gestalt präsentiert, die sich positivem Engagement zu allen gesellschaftlichen Aktivitäten und zum Erfolg zu entziehen scheint und sich nur noch durch das fassen lässt, was sie nicht ist: nicht erwachsen, nichtsnutzig, nicht ordentlich, nicht ehrgeizig, unvermögend im Sinne von arm und nicht könnend, unmöglich, weil er asozial ist. Robert Walsers Räuber ist nur insofern eine Art Held, als seine Kraft oder Macht in Negation der traditionell männlichen Heroik und in der Flucht aus dem Zentrum besteht, und damit der „Existenzweise des Weiblichen“ entspricht, die nach Emmanuel Levinas darin besteht, „sich zu verbergen, und diese Tatsache des Sich-Verbergens ist genau die Schamhaftigkeit" ${ }^{\text {" }}{ }^{6}$

\section{Fazit}

Zum Schluss sei festgehalten, dass die Heldenvorstellung als Ideologie durch ein bestimmtes Medium, in unserer Fallstudie die Literatur, konstruiert und präsentiert wird. Ein literarisches Heldenkonzept hat zwangsläufig eine kulturelle, gesellschaftliche und zeitspezifische Prägung. Sowohl Karl Moor in Friedrich Schillers Schauspiel Die Räuber als auch die Räuber vom Liangschan-Moor in Shuibu Zbuan folgen einem gemeinsamen Heldenkonzept, das je nach den kulturellen und nationalen Vorbildern konstruiert ist.

Als Rebellen lehnen sich die Räuber bei Friedrich Schiller und Shi Naian gegen den vorgefundenen Status quo auf und scheuen nicht davor zurück, Veränderungen ihrer eigenen Schicksale mit Gewalt herbeizuführen. Mag ihre Motivation und ihre Legitimation noch so unterschiedlich erscheinen, bleiben ihre Heldenideen im Grunde genommen vergleichbar. Brüderlichkeit, Maskulinität und Kampfbereitschaft sind Merkmale ihres Heldentums. Einerseits lehnt es sich gegen ein herrschendes System des Unrechts, der Korruption, der Intrigen und der Willkür auf, andererseits ist es auch von einer narzisstischen Disposition und Ich-Bezogenheit geprägt. Die Grundideen des rebellischen Heldentums in den beiden literarischen Meisterwerken bestehen in der radikalen Auseinandersetzung des Individuums mit

45 Walser: Räuber-Roman (Anm. 37), S. 112-113.

46 Emmanuel Levinas: Die Zeit und der Andere, Hamburg 1989, S. 58. 
der Gesellschaft. Sie ist sowohl typisch für die Epoche Sturm und Drang, als auch typisch für die Gesellschaftskonstellation der Song-Dynastie im Reich der Mitte. Dieses Heldenkonzept wird aber in Robert Walsers Romanfragment Räuber radikal dekonstruiert, indem ein postmoderner Protagonist „Räuber“ als negativer Held gestaltet ist, der auf Tapferkeit, Gewalt, Männlichkeit und Siegerlorbeer verzichtet. Alle Wertvorstellungen des herkömmlichen Heldentums werden in Robert Walsers Räuber-Roman auf den Kopf gestellt. Stattdessen wird ein Held ,in Schürze' gepriesen: Weiblichkeit und Zärtlichkeit besiegen wie das Wasser den härtesten Stein. 